\title{
CIENCIA Y TECNOLOGÍA EN EL ECUADOR. UNA MIRADA RETROSPECTIVA HACIA EL FUTURO
}

\author{
SCIENCE AND TECHNOLOGY OF ECUADOR. \\ A RETROSPECTIVE LOOK INTO THE FUTURE \\ Danny Zambrano Veral, Giovanni Herrera Enriquez², \\ Eddy Castillo Montesdeoca ${ }^{3}$, Sergio Castillo Páez $z^{4}$, Pablo Ospina Peralta
}

\begin{abstract}
Resumen
El Ecuador, en la última década, presenta cambios en la estructura y funcionamiento del Sistema Nacional de Educación Superior, nos referimos especificamente al despliegue de proyectos en ciencia, investigación y desarrollo, tales como: Programa Prometeo, Programa Ingenios, Becas de grado y posgrado en universidades nacionales y extranjeras, construcción de cuatro universidades emblemáticas, entre otras iniciativas de innovación y tecnología. Los esfuerzos del Gobierno Nacional durante este periodo fueron diversos para la implementación de ideas de corte social en educación superior, sin embargo los resultados mostraron un sistema parcializado, burocratizado y sin lineamientos en la creación de valor y sinergias entre los diferentes actores participes. El propósito del presente estudio es elaborar un diagnóstico de la situación del Sistema Nacional de Educación Superior de Ecuador en aquellos aspectos inherentes a investigación y desarrollo, ciencia y tecnología, periodo 2012-2014. Para ello se utilizó como instrumento de análisis la Encuesta Nacional de Actividades de Ciencia, Tecnología e Innovación (ACTI), cuyos resultados se exponen a partir del uso de técnicas estadisticas descriptivas, logrando entrever que la asignación incremental de recursos para la educación superior no permitió al final articular una política pública de transformación social, más aún, cuando la rendición de cuentas dejó al descubierto la inconformidad entre sus actores, ahondando la brecha entre sociedad y conocimiento.
\end{abstract}

\section{Palabras clave}

Educación superior, ciencia y tecnología, innovación, sociedad, conocimiento

Clasificación JEL: $\bigcirc 33$

\section{Abstract}

In the last decade, Ecuador present changes in the structure and functioning of the National System of Higher Education, referring specifically to the deployment of projects in science, research and development, such as Prometheus Program, Ingenious Program, Graduate and Postgraduate Scholarships in national and foreign universities, construction of four emblematic universities, among other innovation and technology initiatives. The efforts of the National Government in this period were diverse for the implementation of social ideas in higher education, however, the results showed a biased system, bureaucratized and without guidelines in the creation of value and synergies among the different participating actors. The purpose of this study is to prepare a diagnostic the situation of the National System of Higher Education of Ecuador in those aspects related to research and development, science and technology, period 2012-2014. To this end, the National Survey of Science, Technology and Innovation Activities (ACTI) was used as an analytical tool, which results are exposed from the use of descriptive statistical techniques, managing to see that the incremental allocation of resources for higher education did not allow to articulate of a public policy of social transformation, moreover, when the rendering of accounts reveals the dissatisfaction among its actors, widens the gap between society and knowledge.

\section{Keywords}

Higher education, science and technology, innovation, society, knowledge

JEL Classification: 033

$1 \quad$ Universidad de las Fuerzas Armadas ESPE (Ecuador). C. Económicas, Administrativas y de Comercio. dizambrano@espe.edu.ec 2 Universidad de las Fuerzas Armadas ESPE (Ecuador). C. Económicas, Administrativas y de Comercio. gpherrera@espe.edu.ec. 3 Universidad de las Fuerzas Armadas ESPE (Ecuador). C. Económicas, Administrativas y de Comercio. eacastillo@espe.edu.ec. $4 \quad$ Universidad de las Fuerzas Armadas ESPE (Ecuador). Departamento de Ciencias Exactas. sacastillo@espe.edu.ec.

$5 \quad$ Universidad Andina Simón Bolívar (Ecuador). Departamento del Área de Estudios Sociales y Globales. pablo.ospina@uasb.gob.ec. 


\section{Introducción}

La economía mundial mantiene en alerta a los gobiernos, más aún, cuando los Estados presentan una mayor participación en las decisiones económicas y sociales en una determinada región o territorio. Un aspecto inigualable que preocupa a los gobernantes es precisamente la educación y junto a esta, la articulación de políticas públicas conducentes a la construcción de procesos para el desarrollo, basados en la ciencia, tecnología, investigación y transferencia de conocimientos.

Las políticas de Ciencia, Tecnología e Innovación buscan potenciar los procesos de gobernabilidad consecuentes hacia una investigación proponente y que impulse el desarrollo económico y social de una nación, cuyo eje transversal es la mejora de las condiciones de vida de la población. Siendo el ser humano el elemento central en toda economía, los mecanismos estatistas de intervención deben orientarse hacia la participación individual y colectiva de la persona, e incorporar las condiciones naturales y culturales del ambiente. Surge así un nuevo concepto llamado nodo o polo de desarrollo, el cual es capaz de impulsar las potencialidades de cada territorio, a partir de la integración de las vocaciones productivas de la persona con la naturaleza, la vida y la sociedad (SENPLADES, 2013).

Latinoamérica no es ajena a estos procesos de cambio, más todavía cuando emerge como modelo sui géneris, el gestionar el conocimiento y la tecnología con visión social, lo que pensadores y tratadistas denominan "economía basada en el conocimiento" o "economía del conocimiento" (SENPLADES, 2013, pág. 83). Este modelo mantuvo su presencia en los años ochenta y noventa (incluso hasta los actuales tiempos), cuando Cuba priorizó el mantener un sistema organizacional coherente y sostenible, a partir de la planificación, organización y puesta en marcha de los procesos en investigación científica y tecnológica bajo el enfoque la colaboración multisectorial (Lage, 2013).

Ecuador, partícipe de la propuesta renovada en educación para Latinoamérica, en la última década ha montado una infraestructura política y económica convergente hacia la construcción de una 'sociedad del conocimiento', y que dentro del despliegue forzaría la creación de la 'ciudad del conocimiento'; convirtiéndose esta última en el eje articulador de la estrategia 'cambio de la matriz productiva' (Villavicencio, 2014, pág. 32: 33). Sin embargo, para expertos y académicos, el modelo presentó una dialéctica discursiva frente a la existencia de un pensamiento crítico, regulado por sistemas de indicadores cuantitativos que reducen la calidad y la especificidad de conocimientos localmente significativos, atentando a la satisfacción de las necesidades sociales (Ospina, 2016).

El Sistema Nacional de Ciencia y Tecnología del Ecuador presenta como uno de sus desafíos el acortar las brechas del desarrollo entre los diferentes tipos de economías. Asimismo, la investigación y desarrollo, y la innovación tecnológica, procesos estimulados desde los gobiernos central y descentralizados promueven el crecimiento industrial y de la economía, así como la mejora de las condiciones de vida en la población. Cabe mencionar que, los incrementos de productividad provienen, principalmente, por progresos en el factor tecnológico, fruto de los avances en ciencia y tecnología local y la incorporación de capital humano especializado (INEC, 2016).

De esta forma, el rol del Estado frente al desarrollo científico y tecnológico local cumple características como articulador de la política, participativo, y sobre todo, el de socio estratégico; todas estas capaces de 
apuntalar al Sistema de Ciencia y Tecnología, cuyos elementos son conducentes al desarrollo nacional (Dagnino, Thomas, \& Davyt, 1996). Hoy por hoy, estas carácterísticas también se suman como determinantes de la innovación tecnológica y social, cuyos actores partícipes lo constituyen, el individuo o la sociedad civil, la academia, el capital (empresa e inversión extranjera), sumando los esfuerzos del Estado, a fin de acumular un capital intangible. ${ }^{6}$

Por otro lado, uno de los problemas que influyeron (en principio) en las decisiones del Gobierno del presidente Correa fue el incorporar practicas ortodoxas, en materia de educación superior, ciencia y tecnología, similares a las aplicadas (tiempos atrás) en otros países de la región, hecho que ciertos autores lo catalogaron como enfoque de la dependencia, dando paso a la creación de estructuras internas al interior del Estado y sin prever los requerimientos de la sociedad (Blomström \& Ente, 1990). Incluso, más tarde, el propio gobierno, soportado en la madurez de sus estructuras públicas, propondría el nuevo rol de la economía del conocimiento, pasando desde un enfoque de dependencia hacia el neoestructuralismo, siendo este último el responsable de generar ventajas competitivas en ciertas áreas estratégicas (Kay, 1998), a fin de mantener una integración a la economía mundial y de esta forma reducir la vulnerabilidad y dependencia (CEPAL, 1994; 1995).

El improvisar un enfoque de la dependencia conllevó al gobierno a desplegar una ilimitada infraestructura con una desconcertante inversión pública, vista a partir de la proliferación de instituciones tales como: la Secretaría de Educación Superior, Ciencia, Tecnología e Innovación (SENESCYT); el Consejo de Educación Superior (CES); el an- tiguo Consejo de Evaluación, Acredit ación y Aseguramiento de la Calidad de la Educación Superior (CEACES), en la actualidad Consejo de Aseguramiento de la Calidad de la Educación Superior (CACES); así también, se creó una serie de subsecretarías e instituciones conexas a las anteriores; además de la creación de cuatro universidades emblemáticas (YACHAY, IKIAM, UARTES y UNAE); todo esto con el fin de hacer pensar que se había dado un cambio en el sistema de educación superior, que en la realidad discurre cuando observamos políticas y decisiones aisladas que difieren de una acertada política pública en educación superior.

Para la comprensión del sistema científico-tecnológico nacional, el presente estudio incorpora como instrumento de medición y partida a la 'Encuesta Nacional de Actividades de Ciencia, Tecnología e Innovación (ACTI)', la cual permitió la identificación de las principales actividades que se desarrollan en cuanto a Ciencia y Tecnología siendo estas la investigación y desarrollo, formación y especialización de talento humano, difusión del conocimiento e innovación tecnológica, entre los actores partícipes para el desarrollo tecnológico constan el sector público, el sector privado y la sociedad civil.

La estructura del presente artículo consta de cuatro apartados: la Sección II habla acerca del método empleado, y ante la ausencia de literatura específica sobre el tema se realiza un estudio cuantitativo, de tipo descriptivo y correlacional sobre los resultados obtenidos en la encuesta ACTI, con cobertura nacional. La sección III, presenta los principales hallazgos y resultados del estudio. Finalmente, los apartados IV y $\vee$ presentan aquellas conclusiones más relevantes y las referencias bibliográficas utilizadas.

6 El análisis o evaluación de la actividad científica incorpora como elementos determinantes a la producción y por ende a la productividad, esto visto desde la generación de procesos tecnológicos y científicos, y la posterior acumulación e incremento de un capital intangible, llamado conocimiento (Sancho, 2002). 


\section{Metodología}

Al emplear los datos e información que constan en la encuesta ACTI, cabe precisar que dicho instrumento recoge las experiencias en otras economías y países del mundo, tal es el caso de los miembros de la OCDE, los cuales conjuntamente con la UNESCO y otras organizaciones regionales identificaron las normas para la aplicación de las encuestas. Es conveniente indicar también que estas normas responden, principalmente, a manuales metodológicos sobre I+D (Frascati), Recursos Humanos (Canberra), Balanza de Pagos Tecnológicos y Patentes, entre otros; todos estos inherentes a la construcción de indicadores de Ciencia y Tecnología.

Ecuador, en lo que va del siglo XXI solo cuenta con tres procesos inherentes para recabar información actualizada y confiable sobre Ciencia y Tecnología; el primero auspiciado por la Secretaría Nacional de Ciencia y Tecnología (SENACYT, 2009), la cual publicó sobre "Indicadores de Actividades Científicas y Tecnológicas para el período 2003-2007"; el segundo, desarrollado por el Instituto $\mathrm{Na}$ cional de Estadística y Censos (INEC) y la Secretaría Nacional de Educación Superior, Ciencia, Tecnología e Innovación (SENESCYT), año 2013, y que correspondería a la primera
"Encuesta Nacional de Actividades de Ciencia, Tecnología e Innovación (ACTI)", período 2009-2011; y, finalmente, en el año 2015, bajo convenio interinstitucional INEC-SENESCYT, llevaron a cabo la segunda edición de la encuesta ACTI, período 2012-2014.

Partiendo del uso de información que consta en la encuesta ACTI 2012-2014 se plantea un enfoque cuantitativo, debido a que los datos fueron analizados a partir de procedimientos estadísticos descriptivo y correlacional; no sin antes incorporar a las conclusiones finales del presente estudio notas aclaratorias o explicativas, resultado de la revisión exploratoria de documentos y trabajos de investigación publicados por organismos públicos y de la propia academia.

Es procedente aclarar que, la encuesta ACTI (2015) al presentar características de cobertura nacional incorporó el total de universidades y escuelas politécnicas (públicas y privadas), institutos públicos de investigación, entidades del gobierno central, hospitales públicos de docencia y organismos privados (sin fines de (ucro); todas estas instituciones encargadas (total o parcialmente) de llevar a cabo actividades de Ciencia y Tecnología, arrojando una población de 158 entidades (Cuadro 1).

Cuadro 1. Instituciones de Ciencia y Tecnología

\begin{tabular}{|l|c|}
\hline \multicolumn{1}{|c|}{ Descripción } & Número de instituciones \\
\hline Universidades y escuelas politécnicas & 58 \\
\hline Institutos públicos de investigación & 11 \\
\hline Entidades del gobierno central & 30 \\
\hline Hospitales públicos de docencia & 40 \\
\hline Organismos privados (sin fines de lucro) & 19 \\
\hline Total & 158 \\
\hline
\end{tabular}

Fuente: Encuesta Nacional de Actividades de Ciencia y Tecnología (ACTI), INEC (2015).

Finalmente, como elemento importante se incorpora un resumen de las variables contenidas en la encuesta de Ciencia y Tecnología, instrumento empleado para la recolección de datos e información. Entre las variables principales constan: personas físicas dedicadas a Ciencia y Tecnología, número de graduados, gastos ejecutado en $\mathrm{I+D}$, gasto ejecutado en 
otras actividades científicas y tecnológicas, y número de laboratorios (Cuadro 2). Al igual se incorporan variables agregadas tales como, personas equivalentes a jornada completa, número de investigadores, y gastos en Ciencia y Tecnología según sector de financiamiento.

Cuadro 2. Instituciones de Ciencia y Tecnología

\begin{tabular}{|l|l|l|l|}
\hline \multicolumn{1}{|c|}{$\begin{array}{c}\text { Nombre de la } \\
\text { variable }\end{array}$} & \multicolumn{1}{|c|}{ Descripción de la variable } & $\begin{array}{c}\text { Tipo de } \\
\text { variable }\end{array}$ & $\begin{array}{c}\text { Valores } \\
\text { categóricos }\end{array}$ \\
\hline $\begin{array}{l}\text { Personas físicas } \\
\text { dedicadas a Ciencia } \\
\text { y Tecnología }\end{array}$ & $\begin{array}{l}\text { Corresponde al total de personas físicas dedicadas a activida- } \\
\text { des de Ciencia y Tecnología, desagregada por tipo de función, } \\
\text { sexo, tiempo completo y parcial; además el personal: investi- } \\
\text { gadores y becarios de doctorado, desagregados por: discipli- } \\
\text { na científica, grupos de edad y grado académico alcanzado. }\end{array}$ & numérica & ninguna \\
\hline $\begin{array}{l}\text { Número de } \\
\text { graduados }\end{array}$ & $\begin{array}{l}\text { Corresponde al número de graduados, esta información se } \\
\text { recolecta desagregada por disciplina científica y grado aca- } \\
\text { démico alcanzado. }\end{array}$ & numérica \\
\hline $\begin{array}{l}\text { Gasto ejecutado en } \\
\text { I+D }\end{array}$ & $\begin{array}{l}\text { Corresponde al gasto realizado en I+D; además se recolecta } \\
\text { desagregado por: fuente de financiamiento, tipo e investiga- } \\
\text { ción, disciplina científica, objetivo socioeconómico y destino } \\
\text { de los fondos. }\end{array}$ & numérica & ninguna \\
\hline $\begin{array}{l}\text { Gasto ejecutado } \\
\text { en otras activida- } \\
\text { des Científicas y } \\
\text { Tecnológicas }\end{array}$ & $\begin{array}{l}\text { Corresponde al gasto realizado en otras Actividades Cientí- } \\
\text { ficas y Tecnológicas; además se recolecta desagregado por } \\
\text { fuente de financiamiento. }\end{array}$ & $\begin{array}{l}\text { numérica } \\
\text { Corresponde al número de laboratorio dedicados a Investi- } \\
\text { gúmero de } \\
\text { laboratorio y Desarrollo, se desagrega por disciplina científica. }\end{array}$ & numérica \\
\hline
\end{tabular}

Fuente: Encuesta Nacional de Actividades de Ciencia y Tecnología (ACTI), INEC (2015).

\section{Resultados y discusión}

La encuesta ACTI (2015) muestra que la investigación y desarrollo, y las actividades de ciencia, tecnología e innovación en Ecuador, presentaron aportes e incrementos económicos significativos en el período 2012-2014, tanto del gobierno central como por parte de otras unidades o dependencias (públicas y privadas), alcanzando una media de $850 \mathrm{mi}-$ llones de dólares y una tasa de crecimiento anual de $24 \%$. Sin embargo, en términos de contribución per cápita respecto al PIB, los resultados son nada alentadores puesto que, ni siquiera alcanzan el $1 \%$ en cada uno de los años, exceptuando el 2014 (Tabla 1).

Tabla 1. Gasto en I+D y Actividades de Ciencia y Tecnología como porcentaje del PIB

\begin{tabular}{|l|c|c|c|}
\hline \multicolumn{1}{|c|}{ Gasto } & $\mathbf{2 0 1 2}$ & $\mathbf{2 0 1 3}$ & $\mathbf{2 0 1 4}$ \\
\hline I+D (Investigación y Desarrollo) & $0.33 \%$ & $0.38 \%$ & $0.44 \%$ \\
\hline ACT (Actividades de Ciencia y Tecnología) & $0.42 \%$ & $0.55 \%$ & $0.56 \%$ \\
\hline
\end{tabular}


En cuanto a la participación del gasto en I+D y Actividades de Ciencias Tecnología e Innovación, respecto al PIB observamos que I+D ha pasado de $0.33 \%$ en 2012 a 0.44 $\%$ en 2014, mientras que otras Actividades de Ciencia y Tecnología evidenció un com- portamiento irregular, ubicándose su participación en el 0.12 \% al 2014, al igual que Otras Actividades de Innovación, registrando un $1.32 \%$; incluso está última se convirtió en la actividad de mayor participación durante el período 2012-2014 (Figura 1).

Figura 1. Porcentaje del gasto ACTI con relación al PIB

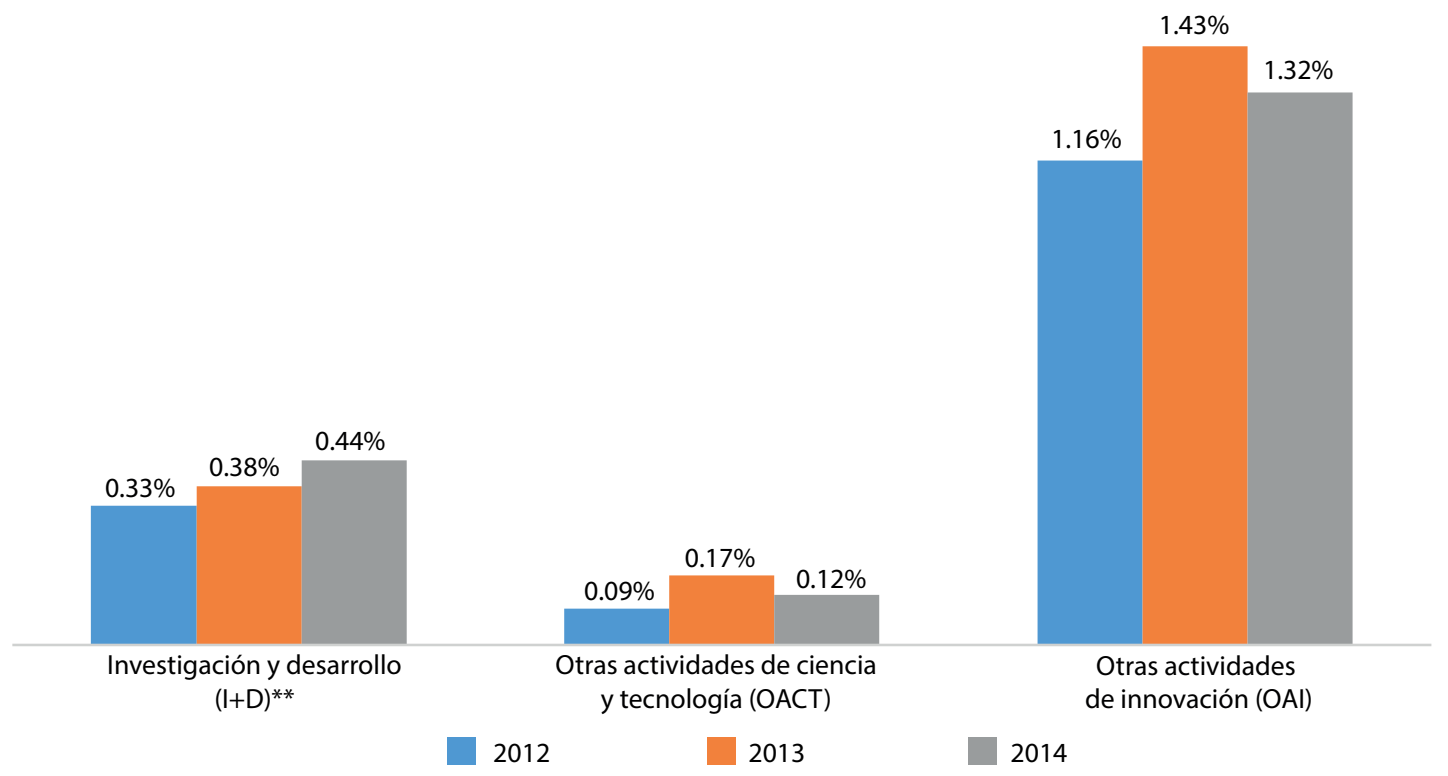

Fuente: Encuesta Nacional de Actividades de Ciencia y Tecnología (ACTI), INEC (2015).

Dentro del mismo análisis de composición económica observamos que el gasto en I+D y Actividades de Ciencia y Tecnología 2012, y según el tipo de investigación, presentó la siguiente distribución: Básica 23.68 $\%$, Aplicada $58.76 \%$ y Desarrollo Experimental $17.56 \%$; al contrario, en los próximos dos años (2013 y 2014) se evidencian comportamientos dispares, donde la investigación básica presenta una tasa de decrecimiento de $9 \%$, a diferencia de la Aplicada y el Desarrollo Experimental que mantienen una tasa de crecimiento anual de $2.6 \%$ (Tabla 2).

Tabla 2. Gasto en I+D según tipo de investigación (Expresado en porcentaje)

\begin{tabular}{|l|c|c|c|}
\hline \multicolumn{1}{|c|}{ Tipo de investigación } & $\mathbf{2 0 1 2}$ & $\mathbf{2 0 1 3}$ & $\mathbf{2 0 1 4}$ \\
\hline Investigación Básica & 23.68 & 18.32 & 19.55 \\
\hline Investigación Aplicada & 58.76 & 66.06 & 61.95 \\
\hline Desarrollo Experimental & 17.56 & 15.61 & 18.50 \\
\hline Total & 100 & 100 & 100 \\
\hline
\end{tabular}

Fuente: Encuesta Nacional de Actividades de Ciencia y Tecnología (ACTI), INEC (2015). 
Por otro lado, los sectores que financian el gasto en I+D y Actividades de Ciencia y Tecnología son: el gobierno, las empresas, la educación superior, las ONG y el sector extranjero; cuyo resumen ponderado para el período 2012-2014 consta a continuación: gobierno $70 \%$ y una tasa media de crecimiento anual de $4.5 \%$, educación superior $25 \%$ y una tasa de (28.90\%), Sector extranjero $4.7 \%$ y una tasa $(10.50 \%)$, mientras que los otros sectores suman una participación testimonial en menos del $1 \%$ e incluso con evidencias de decrecimiento (INEC, 2015).

Otro punto importante, en los gastos realizados en $1+D$ y Actividades de Ciencia y Tecnología, según el objetivo socioeconómico, tenemos que durante el período 2012, el sector de Agricultura presentó una participación de $21.43 \%$, seguido de sistemas políticos y sociales, estructuras y procesos de $13.84 \%$ y con un porcentaje promedio de $11.76 \%$ constan los sectores de Salud, Ambiente y Producción y Tecnología Industrial; los demás sectores registraron una participación inferior a $7 \%$. En cambio, para el año 2014, las participaciones en los diferentes sectores de la economía nacional presentan restructuración frente a sus prioridades; así, Agricultura con un $12.92 \%$, Sistemas políticos y sociales, estructuras y procesos con $12.90 \%$, y Salud, Ambiente, Producción y tecnología industrial con una media de $11.44 \%$; además que otros sectores incrementan su participación, entre ellos, cultura, ocio, religión y medios de comunicación 6.42 \%; Energía 4.45 \%; Educación 5.15 \%; y Avance general del conocimiento I+D 6.76 $\%$ (Tabla 3).

Tabla 3. Gasto en I+D según objetivo socioeconómico (en porcentaje)

\begin{tabular}{|l|c|c|c|}
\hline \multicolumn{1}{|c|}{ Objetivo socioeconómico } & $\mathbf{2 0 1 2}$ & $\mathbf{2 0 1 3}$ & $\mathbf{2 0 1 4}$ \\
\hline Agricultura & 21.43 & 15.07 & 12.92 \\
\hline Sistemas políticos y sociales, estructuras y procesos & 13.84 & 14.82 & 12.90 \\
\hline Salud & 11.65 & 11.71 & 11.74 \\
\hline Ambiente & 11.67 & 11.69 & 10.60 \\
\hline Producción y tecnología industrial & 11.96 & 7.53 & 11.99 \\
\hline Transporte, telecomunicaciones y otras infraestructuras & 6.18 & 8.31 & 6.98 \\
\hline Exploración y explotación del medio terrestre & 6.16 & 5.63 & 5.08 \\
\hline Cultura, ocio, religión y medios de comunicación & 4.29 & 5.69 & 6.42 \\
\hline Energía & 3.44 & 7.08 & 4.45 \\
\hline Educación & 3.92 & 4.28 & 5.15 \\
\hline $\begin{array}{l}\text { Avance general del conocimiento I+D financiada con los fondos generales de } \\
\text { universidades (FGU) }\end{array}$ & 2.63 & 3.06 & 6.76 \\
\hline Defensa & 1.34 & 2.63 & 2.41 \\
\hline Avance general del conocimiento I+D financiada con otras fuentes & 0.90 & 1.93 & 1.96 \\
\hline Exploración y explotación del espacio & 0.60 & 0.55 & 0.66 \\
\hline Total & 100 & 100 & 100 \\
\hline
\end{tabular}

Fuente: Encuesta Nacional de Actividades de Ciencia y Tecnología (ACTI), INEC (2015).

Continuando con la revisión del gasto en I+D según la disciplina científica tenemos, durante el período 2012, las Ciencias Naturales y Exactas con una participación de 26.58
$\%$, Ingeniería y Tecnología $24.80 \%$, Ciencias Sociales $22.36 \%$, Ciencias Agrícolas 19.32 $\%$, mientras que las otras disciplinas (Ciencias Sociales y Humanidades) con apenas 
una participación total de $7 \%$. No obstante, para el 2014, las disciplinas en su gran mayoría presentan comportamientos crecientes en su participación, exceptuado Ciencias
Naturales y Exactas, y Ciencias Agrícolas que presentaron niveles decrecientes, alcanzando $22.64 \%$ y $11.70 \%$ respectivamente (Tabla 4).

Tabla 4. Gasto en I+D según disciplina científica (Expresado en porcentaje)

\begin{tabular}{|l|l|l|l|}
\hline \multicolumn{1}{|c|}{ Disciplina científica } & \multicolumn{1}{c|}{$\mathbf{2 0 1 2}$} & \multicolumn{1}{c|}{$\mathbf{2 0 1 3}$} & \multicolumn{1}{c|}{$\mathbf{2 0 1 4}$} \\
\hline Ciencias Naturales y Exactas & 26.58 & 22.21 & 22.64 \\
\hline Ingeniería y Tecnología & 24.80 & 28.55 & 29.78 \\
\hline Ciencias Médicas & 22.36 & 24.08 & 23.87 \\
\hline Ciencias Agrícolas & 19.32 & 13.51 & 11.70 \\
\hline Ciencias Sociales & 5.01 & 7.65 & 8.13 \\
\hline Humanidades & 1.92 & 4.00 & 3.87 \\
\hline Total & 100 & 100 & 100 \\
\hline
\end{tabular}

Fuente: Encuesta Nacional de Actividades de Ciencia y Tecnología (ACTI), INEC (2015).

Dentro del análisis "Personal de ciencia y tecnología", tomando como base a la jornada completa (EJC) tenemos que para el 2012 existían 3898 investigadores, valor que representa el 0.65 de investigadores por cada mil integrantes de la PEA, de estos investigadores el $70.88 \%$ están localizados en la educación superior, el $27.71 \%$ en el gobierno y solo el 1.41 \% en ONG. Para el 2014, el número de investigadores se incrementó a 5531, cifra que representaba el 0.89 de investigadores por cada mil integrantes de la PEA, y de estos, el 70.87 \% están en educación superior, el $28.08 \%$ en el gobierno, y el $1.05 \%$ en $0 N G$, prácticamente se mantiene la estructura a lo largo del período 20122014 (INEC, 2015).

El limitado número de investigadores, tal cual lo refleja la distribución del párrafo anterior y que fue también evidenciado en el informe de la CEPAL (2016) precisa que, las Ciencias Sociales concentran el mayor porcentaje de investigadores durante el período de análisis, incluso incrementando su participación de $32.01 \%$ en 2012 a $34.07 \%$ en 2014; seguido de las Ciencias Naturales y Exactas de $22.53 \%$ en 2012 a $18.71 \%$ en 2014; y en tercera posición, al igual con un nivel de participación relevante, consta Ingeniería y Tecnología con $17.20 \%$ en 2012 y $20.43 \%$ en 2014 , esta última compensando el decrecimiento que presentó Ciencias $\mathrm{Na}$ turales en el periodo 2014. Las demás disciplinas científicas mantuvieron participaciones por debajo del $13 \%$, incluso al 2014 evidenciando reducciones y ubicándolas en menos del $11 \%$ (Figura 2).

El nivel de formación de los investigadores para el año 2012 presenta la siguiente distribución: $46.01 \%$ maestrías, 39.79 \% tercer nivel, $7.52 \%$ doctorados y $6.68 \%$ Especialistas. Para el 2014, la distribución presenta ciertos cambios, así, maestría alcanza el $47.02 \%$, mientras que tercer nivel disminuyo a $32.06 \%$, al igual que los especialistas a $6.33 \%$, sin embargo el porcentaje de los doctorados incrementa casi al doble, con un total de $14.59 \%$ (INEC, 2015). Estos datos revelan que el gasto destinado en los diferentes niveles de formación corresponde, en su mayoría para maestrías y tercer nivel, con claros signos de crecimiento a nivel de doctorado; esto último como parte de la propuesta de mejoramiento de la educación superior impulsada desde el año 2011. 
Figura 2. Investigadores según disciplina científica en función del total de investigadores

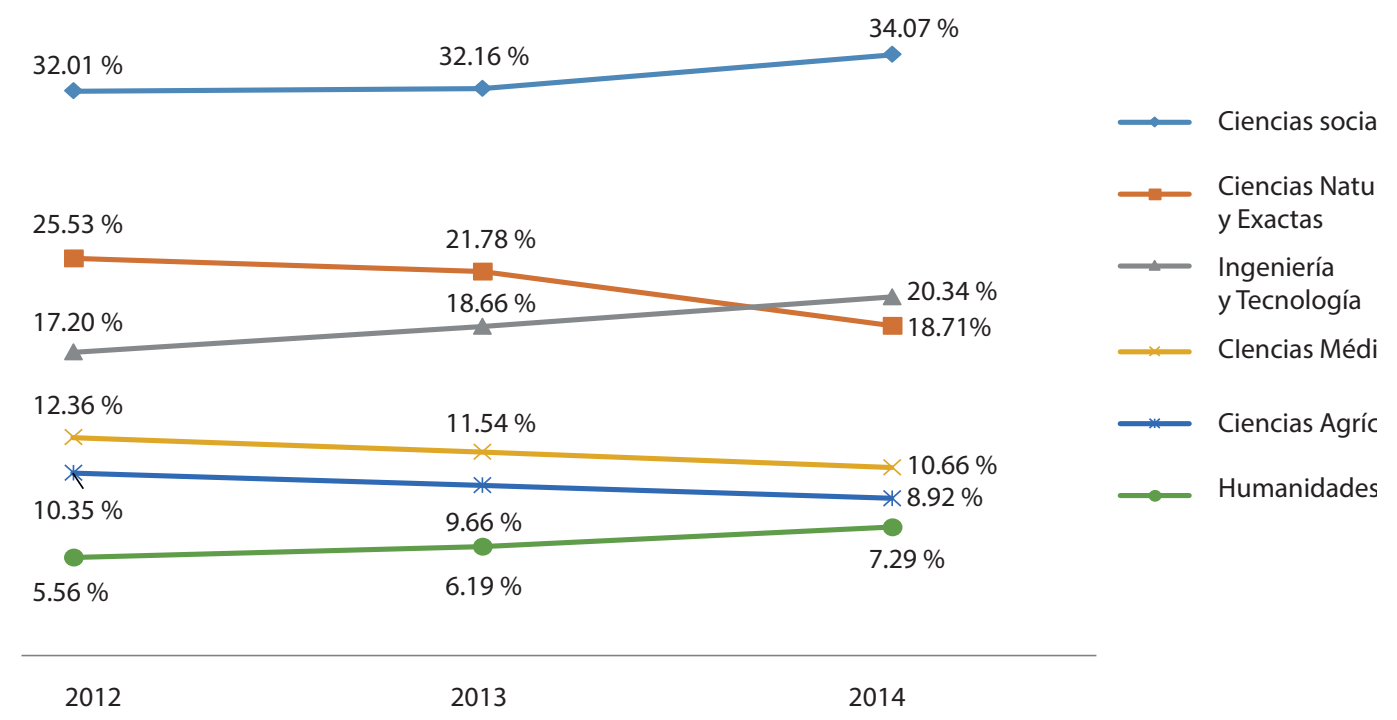

Fuente: Encuesta Nacional de Actividades de Ciencia y Tecnología (ACTI), INEC (2015).

De los graduados en maestrías, se puede evidenciar que Ecuador aún mantiene una alta concentración de sus profesionales en carreras relacionadas a Ciencias Sociales con $54 \%$, mientras que otras disciplinas como Ingeniería y Tecnología, y Ciencias Médicas poseen únicamente el 16 \% y 15 \% respectivamente, a diferencia de las demás disciplinas que presentan una participación conjunta de $15 \%$ (Figura 3).

Figura 3. Graduados de tercer nivel según disciplina científica

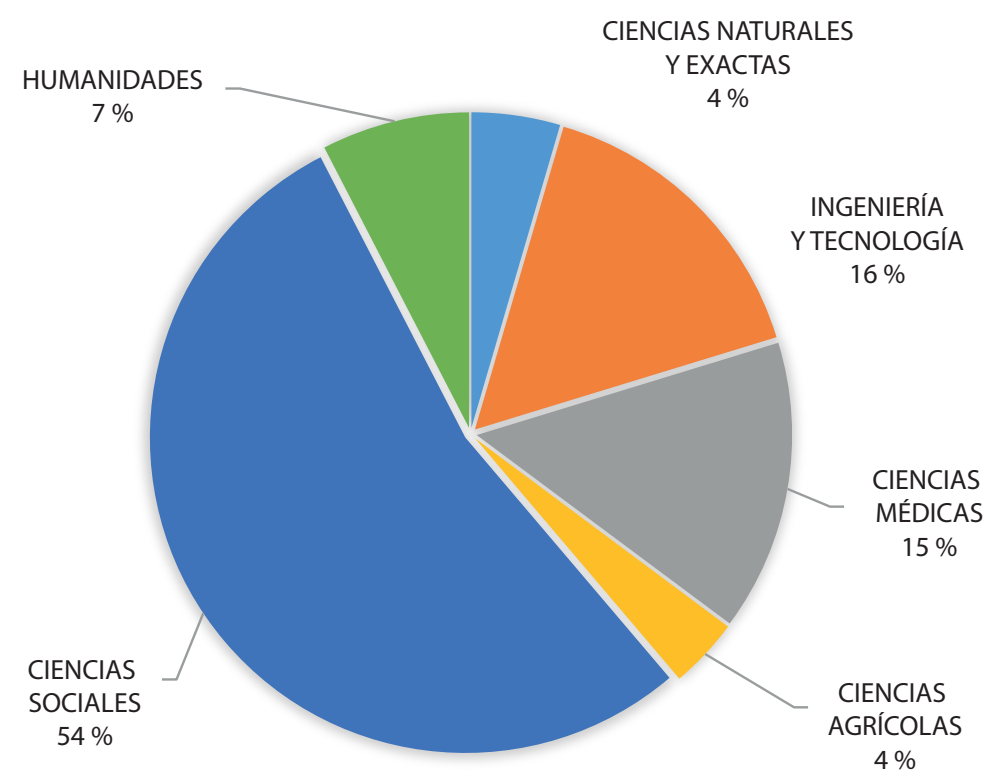

Fuente: Encuesta Nacional de Actividades de Ciencia y Tecnología (ACTI), INEC (2015). 
Finalmente, la formación de posgrado, presenta un mayor predominio de programas relacionados con las Ciencias Sociales (66\%), seguido de Ingeniería y Tecnología y Ciencias Médicas con una participación individual de 10 \%; mientras que las demás áreas (Ciencias
Naturales y Exactas, Ciencias Agrícolas y Humanidades) apenas representan un total de $14 \%$ (Figura 4). Algo muy parecido presentan los otros tipos de programas de posgrado, especialidades y doctorados, donde las Ciencias Sociales concentran un mayor porcentaje.

Figura 4. Graduados de maestría según disciplina científica

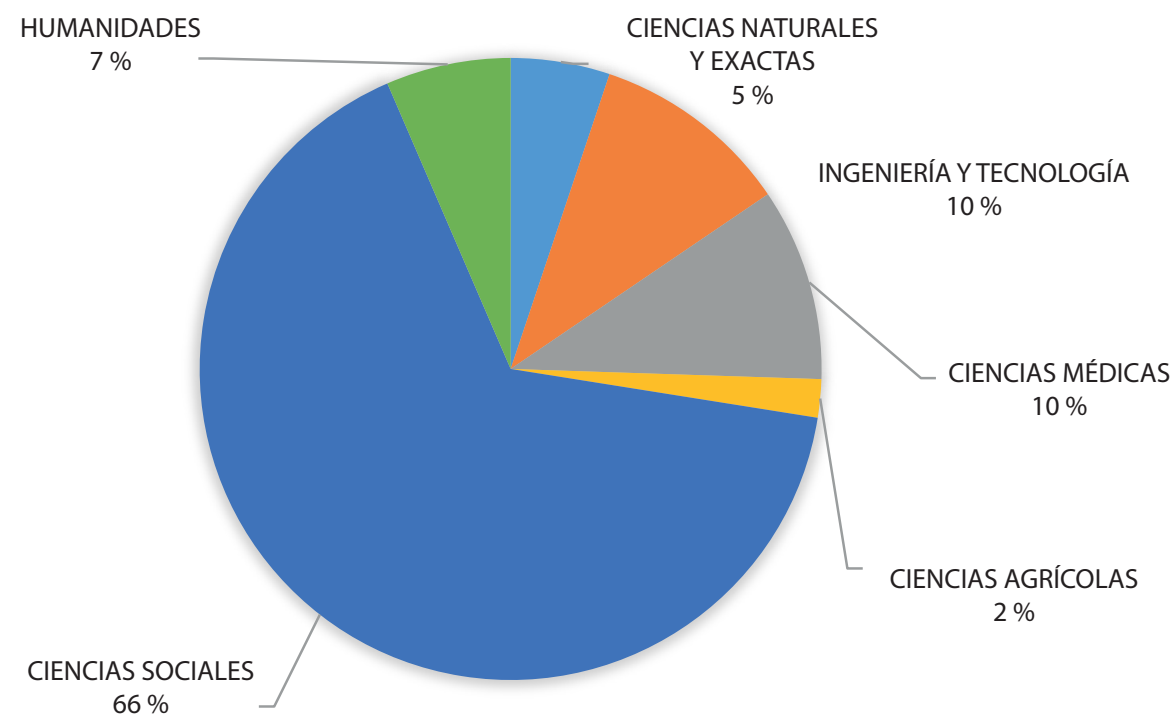

Fuente: Encuesta Nacional de Actividades de Ciencia y Tecnología (ACTI), INEC (2015).

\section{Conclusiones}

La asignación de recursos para las actividades de Ciencia, Tecnología e Innovación, si bien presentaron incrementos durante el período 2012-2014, pasando de 167 millones en el 2012 a 190 millones en el 2014; sin embargo, estos esfuerzos continúan siendo limitados y dispersos; más aún, cuando la participación en gastos de Investigación Básica se redujeron al 19,55\%, mientras que la Investigación Aplicada y el Desarrollo Experimental incrementaron su participación a $61.95 \%$ y $18.50 \%$ respectivamente.

Los sectores que financian la I+D y las Actividades de Ciencia y Tecnología son en mayor porcentaje el gobierno (que incluye todas aquellas instituciones, unidades, empresas de carácter público y mixto) con 70 $\%$, seguido de la Educación Superior con 25 $\%$ y Sector Extranjero con $4.7 \%$; mientras que los demás sectores (empresa privada y ONG) apenas representan el $1 \%$; dejando entrever el papel que cumple el gobierno nacional y en sí, el Sistema de Educación Superior frente a los desafíos de Investigación, Desarrollo e Innovación. De esto último, lo ratifica el Programa de becas y el Proyecto Prometeo, impulsados por el gobierno, lo que ha permitido el desarrollo y fortalecimiento de competencias en investigación científica e innovación tecnológica. 
Todos los esfuerzos señalados, en actividades de investigación, desarrollo e innovación tecnológica, no solo que viabilizaron la identificación de políticas (aisladas y parcializadas) para la educación superior; sino que también aportaron, en cierta medida, a la reducción de la brecha tecnológica, la percepción de un sistema de educación superior excluyente, el bajo nivel de productividad y la asimilación tecnológica. Todo esto, traduciéndose en el principio hacia la construcción del sistema de competitividad nacional limitado o parcializado.

El Gobierno Nacional actual, entre su quehacer económico y social, lleva a cabo un proceso de Reforma Democrática del Estado, cuyos ejes estructurales prioritarios son educación, salud y bienestar social; siendo así, y refiriéndonos en especial a la educación, toda vez que la propia Constitución Política del Ecuador establece que, es un derecho de carácter universal que

\section{Referencias}

Asamblea Constituyente del Ecuador. (2008). Constitución Política del Ecuador 2008. Recuperado el 20 de marzo de 2019 de http://biblioteca.espe.edu.ec/upload/ 2008.pdf

Blomström, M., \& Ente, B. (1990). La teoría del desarrollo en transición. CDMX, México DF: Fondo de Cultura Económica.

CEPAL. (1994). El regionalismo abierto en América Latina y el Caribe: la integración al servicio de la transformación productiva con equidad. Santiago, Chile: CEPAL.

CEPAL. (1995). Latin America and the Caribbean: Policies to Improve Linkages with the Global Economy. Santiago, Chile: CEPAL.

CEPAL. (2016). Ciencia, tecnología e innovación en la economia digital. Santiago, Chile: CEPAL.

Dagnino, R., Thomas, H., \& Davyt, A. (1996). El pensamiento en ciencia, tecnología y sociedad en Latinoamérica: Una interpretación política de su trayectoria. REDES, 3(7), 13-51. garantiza el desarrollo holístico de la nación (Asamblea Constituyente del Ecuador, 2008), este debe interactuar con otros sistemas y agentes de la economía y sociedad, donde la investigación e innovación tecnológica y social se convierten en recursos de desarrollo sostenible.

Ecuador, junto con otros países de la Región (Latinoamérica), en sus planes y programas a mediano y largo plazo prevé impulsar políticas públicas que contribuyan hacia una mejor educación, concomitantemente sea esta capaz de impulsar y mejorar los procesos de desarrollo e innovación (SENPLADES, 2013). En tal virtud, el propiciar e implementar decisiones de investigación al interior de la academia, con enfoque cientista (tecnológico y social), permitirá al propio Estado, otros gobiernos latinoamericanos, y en si la propia sociedad del conocimiento, fijar nuevos objetivos y desafíos que viabilicen el desarrollo sostenible a escala nacional y extraterritorial.

INEC. (2015). Encuesta Nacional de Ciencia y Tecnología 2012-2014, Convenio INECSENESCYT. Instituto Nacional de Estadística y Censos. Recuperado el 10 de junio de 2017, de Ecuador en cifras: http://www. ecuadorencifras.gob.ec/ciencia-tecnologia-e-innovacion-2/

INEC. (2016). Encuesta Nacional de Actividades de Ciencia y Tecnología (ACT): 2012-2014. Quito, Ecuador: INEC.

Kay, C. (1998). Estructuralismo y teoría de la dependencia en el período neoliberal. Una perspectiva latinoamericana. Nueva Sociedad(158), 100-119.

Lage, A. (2013). La economá del conocimiento y el socialismo. La Habana, Cuba: Sello Editorial Academia.

Ospina, P. (2016). Política universitaria: espejo político del correísmo. Recuperado el 24 de Noviembre de 2017 de Nueva Sociedad: Democracia y política en América Latina: http:// nuso.org/articulo/politica-universitaria-espejo-politico-del-correismo/ 
Sancho, R. (2002). Indicadores de los Sistemas de Ciencias, Tecnologia e Innovación. Economía Industrial(344). Recuperado de http:// www.minetur.gob.es/Publicaciones/ Publicacionesperiodicas/Economialndus trial/RevistaEconomialndustrial/343/ 097-SANCHO.pdf
SENPLADES. (2013). Plan Nacional del Buen Vivir 2013-2017. Quito: SENPLADES. Recuperado el 11 de Diciembre de 2017, de http://www. buenvivir.gob.ec/planificamos-el-futuro

Villavicencio, A. (2014). Innovación, matriz productiva y universidad: porque Yachay es una estrategia equivocada. Quito: Fundación Hernán Malo / Corporación Editora Nacional. 